INGURUAK [69] | 2020 | 39-56

https://doi.org/10.18543/inguruak-69-2020-art03

ISSN 0214-7912

\title{
Donde se sostiene la vida: cuidados, cuerpo(s) y comunidad en tiempos de pandemia
} Where life is sustained: care, body (s) and community in times of pandemic

Inés González Celis*, Amaia González Llama

Universidad del País Vasco / Euskal Herriko Unibertsitatea

RESUMEN: La extensión mundial de la COVID-19 y su consecuente crisis sanitaria han sacado a la luz desigualdades sociales presentes pero ocultas desde hace años, entre ellas la crisis de los cuidados, que aquí analizamos. Para entender los motivos por los que el contexto actual de pandemia ha afectado de forma tan profunda a la esfera de los cuidados es necesario comenzar por un acercamiento al papel que ocupan en la sociedad, ahondando en las causas y consecuencias de su invisibilización. Para ello, dado que los cuidados siempre se realizan desde y hacia el cuerpo, es necesario profundizar en el significado de este. El análisis sociológico del concepto «cuerpo» nos introduce en el cuestionamiento sobre la responsabilidad de las ciencias sociales en su invisibilización y, al mismo tiempo, sobre su utilidad para generar cambio social desde un cambio de perspectiva del mismo concepto. Finalmente, un acercamiento a las distintas iniciativas sociales que se han llevado a cabo contra la pandemia, y que presenta el lado más esperanzador de esta crisis, nos muestra la importancia de la organización comunitaria en la toma de acción para paliar los efectos de las profundas desigualdades sociales actuales.

Palabras clave: cuidados, cuerpo, COVID-19, crisis, comunidad.

ABSTRACT: The global spread of COVID-19 and its consequent health crisis has brought to light social inequalities that have been present but hidden for years, including the care crisis, which we analyse here. In order to understand why the current pandemic context has affected the care sector so profoundly, it is necessary to start by looking at the role of care in society, delving into the causes and consequences of its invisibility. To this end, given that care is always provided from and to the body, it is necessary to delve deeper into the meaning of the body. The sociological analysis of the concept «body" introduces us to the questioning of the responsibility of social sciences in its invisibility and, at the same time, of its usefulness in generating social change from a change of perspective of the same concept. Finally, a look at the different social initiatives against the pandemic that have been carried out, which presents the most hopeful side of this crisis, shows us the importance of community organization in taking action to alleviate the effects of the deep social inequalities of today.

Keywords: care, body, COVID-19, crisis, community.

* Correspondencia a/Correspondence to: Inés González Celis. Universidad del País Vasco/Euskal Herriko Unibertsitatea - inesgc659@gmail.com

Cómo citar/How to cite: González Celis, Inés; González Llama, Amaia (2020). "Donde se sostiene la vida: cuidados, cuerpo(s) y comunidad en tiempos de pandemia»; Inguruak, 69, 39-56. (https://doi.org/10.1387/inguruak-69-2020-art03).

Recibido/Received: 28 setiembre, 2020; Versión final/Final version: 25 noviembre, 2020.

ISSN 0214-7912 / (C) 2020 UPV/EHU

(C) (B) Esta obra está bajo una licencia

Creative Commons Atribution 4.0 Internacional License 


\section{INTRODUCCIÓN}

Los cuidados mueven el mundo, lo hacen hoy y lo han hecho siempre. Desde que sostenemos la cabeza de la recién nacida hasta que sostenemos la mano de la anciana en sus últimos momentos, vivimos en un ciclo que solo es posible gracias al amor y la colaboración de nuestras semejantes. Hoy, un virus llamado COVID-19 nos ha colocado frente a esta realidad obligándonos a asumir que somos seres interdependientes y vulnerables, ayudándonos a entender que la vida sin comunidad no tiene futuro. Desde que la pandemia se hizo oficial y el confinamiento fue una realidad, innumerables problemáticas sociales han visto la luz, la mayoría de ellas previamente denunciadas. Este trabajo analiza la relación entre estas problemáticas y la denominada crisis de los cuidados, una de las más significativas en relación a lo que las políticas neoliberales imperantes representan, ya que muestra su desprecio al elemento central de la existencia: la corporalidad. Nos situamos en el marco del Estado Español, aunque centrando la mayoría de ejemplos concretos en el caso de la Comunidad Autónoma Vasca.

A continuación, y antes de comenzar con nuestro análisis, realizamos un breve repaso de lo sucedido desde la declaración oficial de la pandemia. El 14 de marzo el Consejo de Ministros aprueba la declaración de Estado de Alarma en todo el territorio durante 15 días naturales, con medidas de restricción al movimiento de personas y la actividad económica. Los desplazamientos se limitan a lo imprescindible y comienza el llamado «confinamiento» de la población, con excepción de determinados sectores laborales, con el objetivo de frenar la expansión de la COVID-19, declarada pandemia mundial por la OMS el 11 de marzo. El 28 de marzo, ante la gravedad de las cifras de fallecimientos y contagios, el Gobierno anuncia la paralización de todas las actividades no esenciales, medida que finaliza el 14 de abril con el comienzo del declive en las curvas de afectados y fallecidos.

El 26 de abril se decreta el fin del confinamiento estricto para las niñas y niños y el 28 el Gobierno aprueba un plan de desescalada con cuatro fases y distintas velocidades según islas y provincias, con una duración de entre 6 y 8 semanas. Aunque las prórrogas del Estado de Alarma no cesan hasta el 21 de junio. Una vez finalizado el plan de desescalada y el Estado de Alarma se da comienzo a un periodo denominado «nueva normalidad», cuya base reside en intentar convivir con el virus cambiando lo menos posible la vida previa a la expansión de este, y cuyo fin no se plantea hasta el desarrollo de una vacuna que le haga frente. Tras el verano, ante la continua expansión de la COVID-19 y la ausencia de vacuna, el 25 de octubre se declara de nuevo el Estado de Alarma, con la intención de que este se alargue hasta mayo, y comienzan a implantarse de nuevo medidas restrictivas en todo el territorio desde los gobiernos autonómicos.

Así, desde el inicio de la pandemia y hasta el pasado 4 de noviembre, según datos del Ministerio de Sanidad, para el caso de España, y de la Universidad John Hopkins para el resto 
de países, se habían diagnosticado en todo el mundo 48.107 .322 casos y el número de personas fallecidas alcanzaba la cifra de 1.225.463 víctimas; en España, los casos confirmados tras la realización de pruebas PCR ascendían a 1.284.408 personas y la cifra de muertes era de 38.118 (Clemente et al., 2020). Hay que tener en cuenta, además, que el Sistema de Monitorización de la Mortalidad diaria (MoMo) del Instituto de Salud Carlos III, que registra los datos totales de defunciones diarias notificadas por los registros civiles, sin especificar la causa de muerte, viene señalando desde el inicio de pandemia un importante exceso de mortalidad en España, es decir, un número de fallecimientos muy superior al esperado en condiciones normales, y mayor también que la cifra de muertes por COVID diagnosticadas. A esto hay que añadir que muchas de las personas que han sufrido y superado el coronavirus han resultado gravemente afectadas por diversos efectos secundarios (Del Rio et al., 2020), que en muchos casos supondrán una merma importante en la calidad de vida de las personas afectadas.

En este contexto, comenzamos nuestro análisis con un repaso a la crisis de los cuidados ahondando en sus orígenes e incidiendo en el hecho de que la crisis sanitaria actual no ha hecho sino sacar a la superficie lo que desde hace décadas se viene denunciando (Ezquerra, 2011; Addati et al., 2018; Alabao, 2019; Coffey, 2020). Continuamos con un análisis sociológico del concepto "cuerpo" que nos introduce en el cuestionamiento sobre la responsabilidad de las ciencias sociales en su invisibilización y, al mismo tiempo, sobre su utilidad para generar cambio social desde un cambio de perspectiva del mismo concepto. Por último, nos adentramos en las iniciativas sociales que se han llevado a cabo para combatir las consecuencias de la pandemia sobre las personas y colectivos más vulnerables y que muestran el lado más esperanzador de la actual crisis.

\section{DERECHO A LA VULNERABILIDAD, RECONOCIMIENTO DE LOS CUIDADOS}

Hay cosas que siempre han estado ahí, el piar de los gorriones por las mañanas, las risas del niño que vive enfrente, el viento sobre las hojas..., pero que no hemos percibido hasta que la excepcionalidad traída por el coronavirus ha llegado. La crisis de los cuidados es una de ellas. Amaia Pérez Orozco (2006: 9) sostiene que esta crisis «solo se hace visible si dejamos de poner la mirada en lo económico y empezamos a ponerla en la sostenibilidad de la vida", algo que, en cierto modo, la crisis sanitaria provocada por la COVID-19 nos está obligando a hacer. No es que el interés por la perpetuación de las dinámicas del mercado por encima de todo haya desaparecido, es que ha ocurrido algo que ha desvelado que el correcto funcionamiento del mercado depende directamente del correcto funcionamiento de las personas. La enfermedad pone límites al cuerpo, y cuando esa enfermedad se convierte en lo que podríamos llamar un hecho social total también pone límites al mercado. De repente la mirada se pone en la vida, porque lo contrario es la muerte, no solo de quienes permanecen en los márgenes, sino de quienes siempre han ocupado el centro hegemó- 
nico: la muerte del mercado y del bienestar físico, emocional, mental y económico de todas las personas que lo conforman.

El funcionamiento del sistema económico neoliberal se basa en la concepción de las personas trabajadoras como seres que nacen y crecen libres de cargas o necesidades de cuidados, entendidos meramente como «recursos humanos» para conseguir una mayor acumulación de capital. Esto genera un conflicto entre esa lógica de acumulación y la sostenibilidad de la vida, que es socialmente aceptable porque permanece oculto, inmerso en la esfera doméstica. Así, el funcionamiento del sistema solo es posible en la medida en que esa responsabilidad social que el mercado elude es asumida por las esferas invisibilizadas de la economía. Tras esa idea del individuo autónomo se esconde todo un trabajo de cuidados, encarnado prácticamente en su totalidad por redes de mujeres que al sostener la vida sostienen igualmente toda la actividad económica productiva (Carrasco, 2011, 2013, 2017).

Para comprender de manera clara esta realidad y ser capaces de establecer la relación entre el estado de alarma provocado por la crisis sanitaria y la visibilización del entramado oculto que sostiene la vida, es necesario partir de la identificación de qué hablamos cuando hablamos de cuidar. La idea de los cuidados comienza a estar presente en un gran número de discursos, lo que por una parte lo dota constantemente de significado y de nuevas visiones; pero también puede llevar a una banalización del mismo cuando no tenemos muy claro a qué nos estamos refiriendo exactamente. La falta de un debate a fondo para aclararnos y poner posturas en común lleva en numerosas ocasiones a la reducción de estos a las tareas domésticas (cuidado de niños y mayores, cocina, limpieza...), las cuales son claramente parte del trabajo de cuidados, pero no son las únicas. Citando de nuevo a Pérez Orozco podemos establecer que:

Por cuidados podemos entender la gestión y el mantenimiento cotidiano de la vida y de la salud, la necesidad más básica y diaria que permite la sostenibilidad de la vida. Presenta una doble dimensión «material», corporal — realizar tareas concretas con resultados tangibles, atender al cuerpo y sus necesidades fisiológicas - e «inmaterial», afectivo-relacional — relativa al bienestar emocional-. Cuidar es «hacerse cargo de los cuerpos sexuados» (Precarias a la deriva, 2005b) y de las relaciones que los atraviesan. (Pérez Orozco, 2006: 10)

O, remontándonos a la caracterización formulada en 1990 por Berenice Fischer y Joan Tronto, cabe entender por cuidado:

Una actividad de especie que incluye todo aquello que hacemos para mantener, continuar y reparar nuestro «mundo» de tal forma que podamos vivir en él lo mejor posible. Ese mundo incluye nuestros cuerpos, nuestros seres y nuestro entorno, todo lo cual buscamos para entretejerlo en una red compleja que sustenta la vida. (citado en Tonto, 2005: 234) 
Partiendo de este planteamiento, es importante realizar un análisis que nos lleve a entender a qué se debe la desvalorización de los cuidados. La parte más clara es que estos permanecen a la sombra porque la ocultación de esa dependencia entre la esfera productiva y la reproductiva de la que hablábamos antes permite al mercado una reducción de los costes relacionados con la reproducción de la mano de obra y el mantenimiento de la población. Pero el trasfondo de esta desvalorización va mucho más allá, ubicando sus raíces en una división dicotómica del mundo en la que una parte siempre se sitúa por encima de la otra, y en la que los cuidados se relacionan con la parte oprimida de cada dicotomía. Las tres parejas que consideramos más significativas a la hora de analizar los cuidados son hombre/mujer, mente/cuerpo y público/privado. La lógica de acumulación del capital y la de sostenibilidad de la vida son dos lógicas marcadas por el género, tanto a nivel material como a nivel simbólico por la concepción del mundo que se les atribuye (Pérez Orozco, 2006). El imaginario del mundo se ha construido desde una lógica androcéntrica donde el cuidado del entorno y la comprensión de los propios límites quedan relegados en pos de un crecimiento material ilimitado, situando la mente - lo racional, abstracto- por encima del cuerpo - lo emocional- que se relaciona con la mujer, enmarcando esta centralidad en el espacio público, y ocultando todo lo que muestra vulnerabilidad, y por tanto requiere de cuidado, en el espacio privado.

Es aquí donde entra en juego la importancia de las consecuencias de la declaración del estado de alarma, al establecer una serie de medidas que diluyen la rígida línea que separa espacio público-productivo y espacio privado-reproductivo. Por un lado, el teletrabajo desde el hogar en aquellos empleos en los que este es posible ha significado la irrupción de la esfera pública en la privada que, en consecuencia, se hace más visible que nunca. Sin embargo, la simultaneidad de esto con el cese de la actividad escolar, así como de gran parte de sectores económicos, ha hecho que la imposibilidad de conciliar el empleo remunerado y el trabajo de cuidados se haga aún más patente. Estas medidas devuelven los cuidados exclusivamente a una esfera privada que, aunque como ya hemos señalado se vuelve más visible, sufre tal abandono por parte del Estado que lleva a la búsqueda de soluciones individuales. El cierre de colegios y guarderías, el cese del trabajo doméstico remunerado, el aislamiento de la población envejecida... sacan a la luz una serie de actividades con un enorme valor en términos de tiempo y de calidad de vida a las que el Estado se muestra incapaz de dar respuesta. De pronto, muchas de esas actividades hasta ahora invisibilizadas han empezado a ser codificadas como «esenciales», pero solo en el nivel del discurso. Lo que supone un problema (que ya estaba presente pero que ahora, al incrementar sus dimensiones, se vuelve difícil de ignorar) para todas aquellas personas que tienen a otras a su cargo y deben responder al mismo tiempo a las exigencias del mercado. Esto nos lleva a preguntarnos a quién cuida el Estado, cómo y bajo qué lógicas. Recordemos, en este sentido, que a principios del pasado mes de octubre el Comité Español de Representantes de Personas con Discapacidad (CERMI) denunció el «fracaso» del Sistema de Autonomía y Atención a la Dependencia (SAAD) y exigió al Gobierno la «refundación» de este sistema (CERMI, 2020). Si cuidar también es proteger, vemos de forma clara como el Estado en épocas anteriores de crisis ha cuidado al mercado, guiándose por las lógicas del capital y no de la vida. 
En el marco del Estado de Bienestar, dentro de la Unión Europea existe un interés por crear empleo en el sector de los servicios de cuidados como parte de esas políticas del bienestar. Sin embargo, cuando esos cuidados no pueden ser aportados a través del mercado, como ocurre actualmente, sino que deben ser acompañados a través de ayudas de tiempo y dinero, el papel del Estado parece diluirse por completo. Ignorando así que la dependencia es algo universal y que, por tanto, la responsabilidad de los cuidados que le dan respuesta debería ser social y política (Carrasco et al., 2011). Ante este hecho cabe destacar el reforzamiento del tejido social; nos parece reseñable la forma en que la comunidad se articula de forma espontánea en épocas de crisis para dar una respuesta colectiva a las necesidades que el Estado no es capaz de cubrir.

Igualmente, si partimos de la importancia que supuso la industrialización en la división sexual del trabajo, el cese de toda actividad económica no considerada como necesaria durante varias semanas, nos lleva a preguntarnos de qué forma afecta a la división del trabajo doméstico. Reflexionando principalmente sobre la masculinización de algunas tareas, como hacer la compra, y las lógicas que esto oculta. Cuando poder salir a la calle para comprar se convierte en un privilegio, ¿quién ocupa esos espacios? Recordemos que un estudio de las economistas Lídia Farré y Libertad González realizado en las primeras semanas del confinamiento detectaba que el hombre estaba pasando a ser el principal responsable de la compra (Farré y González, 2020).

Por otro lado, esta crisis también saca a la luz de forma clara el conflicto entre el capital y la vida, pues la fragilidad de la vida se hace más clara que nunca. Hemos visto cómo en plena crisis sanitaria se ha seguido produciendo una especulación económica con material sanitario, lo que tiene efectos directos en la salud de la población. Asimismo, es en esta situación donde se vuelve más sencillo identificar la relación entre las políticas económicas hegemónicas y la sostenibilidad de la vida. Desde la forma en que estamos padeciendo los recortes sanitarios de hace unos años, hasta cómo decisiones relacionadas con el pago de alquileres o ayudas a trabajadoras autónomas (entre otras) tienen efectos en la calidad de vida de las personas, pasando por el olvido de la esfera doméstica a la hora de cancelar las clases sin previo aviso y sin alternativas al cuidado de las y los menores.

Finalmente, es de especial importancia volver a lo ya comentado para resaltar la forma en que la enfermedad hace visible la vulnerabilidad natural del ser humano y las distintas vulnerabilidades provocadas por el sistema. La enfermedad nos despierta de nuestros sueños divinos para devolvernos a la tierra, a lo real, a los límites, a la interdependencia entre las personas. Nos expone a todas a la muerte, mostrando que ni siquiera el mercado tiene la solución para la vida eterna. Vuelve dependientes a quienes creyeron no serlo, porque nos recuerda que la dependencia se vincula con la necesidad, y que esta también se construye socialmente. Además, choca de lleno con la realidad de que no todas somos igual de vulnerables, porque hay vulnerabilidades que son socialmente construidas. La enfermedad y la forma en que se está abordando, vuelve a dejar al margen a quienes ya lo estaban. 
Como nos cuestiona el Grupo de trabajo Ética y Covid Euskadi (2020): «¿La salud de quién estamos defendiendo?».

El confinamiento pone en evidencia la precariedad a la que está sujeta la vida de algunos sectores de la población, profundizando las desigualdades y sacando a la luz la situación de las personas que se enmarcan en situaciones fuera del mercado regulado (limpiadoras del hogar, prostitutas, trabajadoras transfronterizas en Ceuta y Melilla, migrantes, personas sin hogar, drogodependientes, manteros, personas privadas de libertad...). Algo que no es nuevo y que guarda una relación directa con todo lo expuesto anteriormente. La ejemplificación más clara de esta conexión es la situación que atraviesa a las trabajadoras del hogar, principalmente a las migradas: «el sujeto del trabajo de cuidado es un sujeto colectivo; los cuidados son mayoritariamente realizados por redes de mujeres» (Pérez Orozco, 2006: 15), redes que llegan a ser transnacionales, formando lo que llamamos la cadena global de cuidados.

\section{CUERPOS Y PRECARIEDAD}

El cuerpo en relación con el mundo ha sido siempre un objeto de estudio de la sociología y, sin embargo, carecemos de una definición unánime sobre él, sobre su significado y sobre la manera en que debemos tratarlo desde la ciencia social (Oliva Portolés, 2015; Angelcos, 2016; Sáenz et al., 2017). La ambigüedad en relación a su significado en la ciencia social se debe a que este ha sido habitualmente materia implícita y casi nunca explícita del análisis de «las modalidades físicas de la relación del actor con el mundo» (Le Breton, 2018: 52). Este es, al fin y al cabo, el punto neurálgico de las diferentes Sociologías, en especial, de la sociología del trabajo, que hoy cobra protagonismo debido a la profunda crisis que la COVID-19 ha desatado. Simone Weil, en una conferencia ofrecida en 1937, puso de manifiesto que existían dos revoluciones industriales: la que «se define por la utilización científica de la materia inerte y de las fuerzas de la naturaleza [y la que] se define por la utilización científica de la materia viva, es decir, de los hombres» (Weil, 2014: 225). Materia viva, aquello de lo que está hecho el cuerpo, aquello sin lo cual no hay existencia posible, ya que «la existencia es, en primer lugar, corporal» (Le Breton, 2018: 9).

Dejar en manos de la libre interpretación la definición del «cuerpo» pone en peligro la posibilidad de encontrar soluciones adecuadas a los problemas que lo atraviesan. Además, perder de vista este elemento esencial del análisis sociológico responsabiliza a la disciplina de la perpetuación del discurso hegemónico dualista que separa cuerpo y mente y que justifica la alienación de la que ya hablaban en el siglo xix Marx y Engels, entre otros. Son precisamente estos dos autores quienes ilustran el paradigma del estudio de la corporalidad sin la elaboración previa de su definición. Ambos analizaron y denunciaron los efectos de las condiciones laborales en las vidas de las personas de clase trabajadora que es, en todo caso, analizar la incidencia de determinados factores sociales en el cuerpo de los trabaja- 
dores y, al mismo tiempo, estudiar la incidencia que esos cuerpos maltratados tenían en sus diferentes entornos. Sin embargo, al no ser la corporalidad un objeto de estudio explícito, todo su análisis quedó clasificado en temáticas diferentes (trabajo, socialismo...) dificultando a las investigadoras interesadas en el estudio de la corporalidad el acceso a tan vasto conocimiento. Marx, Engels y sus discípulos abordaron la corporalidad sin definirla, y esto fue causa y consecuencia de que quedara en segundo plano. Este desplazamiento de la corporalidad, acompañado y alimentado por la monomanía de la contabilidad (Weil, 2014: 226) imperante desde la Revolución Industrial, ha concluido en que la mayoría de las reivindicaciones de la clase trabajadora se hayan centrado en el precio del trabajo y no en su valor; en los beneficios que la persona empleada debe recibir por lo que produce en relación a los beneficios que la empresaria recibe. La energía, el tiempo, el desgaste o la pérdida del sentido vital que supone el trabajo realizado siempre estuvieron en un segundo plano. De esta manera, es casi una fórmula matemática la que explica que trabajos que no producen beneficios económicos directos, como los relacionados con cuidados, no hayan sido objeto central de lucha, ya que no hay rendimiento económico que reclamar al patrón. Por supuesto, esta fórmula queda incompleta sin los factores "género» y «origen», puesto que el capitalismo, se ha sustentado históricamente en la dominación de las mujeres mediante el patriarcado, y de quienes habitan territorios invadidos mediante el colonialismo.

«El cuerpo es un constructo social [...] [cuya caracterización] lejos de ser unánime en las sociedades humanas, demuestra ser sorprendentemente complicada», recuerda Le Breton (2018: 39); es un conjunto de símbolos cuyas interpretaciones varían con base en la cultura que se habita, y, puesto que es el «vector semántico por medio del cual se construye la evidencia de la relación con el mundo» (Le Breton, 2018: 9), además de recibir estímulos externos, transmite información y modela el entorno.

Somos cuerpo, no tenemos cuerpo, o, más exactamente, "somos cuerpo desde el que emerge un sujeto» (Álvarez Cantalapiedra, 2017: 7). Esta es la idea fundamental que permitirá construir una nueva forma de habitar el mundo que evite que el cuerpo continúe siendo «reducido a mero objeto susceptible de ser comprado o vendido al mejor postor, enajenado, troceado o separado de la persona» (Álvarez Cantalapiedra, 2017: 7). En este punto debemos diferenciar entre corporalidad, entendida como la parte física del ser que «no solo reacciona ante estímulos externos como lo haría la materia inerte ante un cambio de presión o temperatura, sino que ante esos y otros estímulos ejecuta actividades para seguir siendo lo que es, y en el proceso modifica el medio que lo modifica» (Álvarez Cantalapiedra, 2017: 7); y cuerpo-sujeto, entendido como «la sinergia de genes y ambiente, naturaleza y crianza, evolución biológica e influjos culturales y educativos» (Álvarez Cantalapiedra, 2017: 8).

Obviar al cuerpo, por concebirlo como un elemento que se da por hecho, y no como una «falsa evidencia [...] efecto de una elaboración social y cultural» (Le Breton, 2018: 39) lleva inevitablemente a una errónea elaboración de preguntas sobre sus cualidades y, por con- 
siguiente, a respuestas y soluciones separadas de sus necesidades. La pregunta "¿de qué cuerpo hablamos?» se diluye y pierde sentido en un contexto que da por sentada su existencia, e imposibilita el cuestionamiento de los cánones estéticos y de salud hegemónicos. Este es el camino que transitan numerosos estudios sociológicos al plantear la precariedad desde el punto de vista de la relación de las «actoras" y actores con la estructura establecida. Las preguntas acerca de la sensación de precariedad habitualmente están dirigidas a valorar la calidad de vida en relación a la tenencia o no de los elementos establecidos como necesarios. En raras ocasiones estos análisis cuestionan desde el punto de vista de la corporalidad y del cuerpo-sujeto, es decir, del propio ser, si las metas socialmente establecidas contribuyen o no a la construcción de vidas precarias.

El planteamiento de la precariedad desde el estado actual de las cosas es necesario, pero insuficiente. Es necesario para conocer las incoherencias entre lo que la estructura socioeconómica impone a la población como imprescindible para la construcción de vidas dignas, y las posibilidades que sus propias dinámicas económicas y políticas ofrecen para conseguirlo; y es también necesario para observar hasta qué punto las personas han asumido como suyos los objetivos vitales socialmente establecidos. Sin embargo, este tipo de cuestionamiento resulta insuficiente para entender las diferentes percepciones personales sobre lo que es precario y lo que no en relación al propio ser, en relación al «sentido de lo humano» (Antón Hurtado, 2012: 350). La omisión de esta perspectiva en los estudios sociológicos sobre precariedad abre el debate sobre la dimensión ética de los mismos, por no favorecer la posibilidad de crear nuevas demandas que pongan en el centro la corporalidad y, con ello, las necesidades básicas de la existencia, y perpetuar, así, el statu quo: "se escucha, cada vez con más intensidad, la idea de que la actitud acrítica que han venido asumiendo las ciencias sociales bajo la corriente culturalista (Lizón, 2010: 412; Aguiar, Francisco y Noguera, 2009: 443) debe dejar paso a una nueva cultura en la que la persona piense y decida por sí misma y los hechos cobren mayor relevancia que los datos» (Antón Hurtado, 2012: 353). Comenzar a plantearnos la precariedad laboral y vital desde el punto de vista de la consciencia de la corporalidad propia y ajena y de su relación con la propia existencia, en lugar de hacerlo solo desde el punto de vista de lo establecido externamente como necesario para una vida digna, permite la elaboración de demandas sociales desde lo deseable y no solo desde lo posible.

\section{A COMUÑA ${ }^{1}$}

Así se titula un precioso cuento en asturiano de la escritora Marina Lobo (2019), que se publicita con esta hermosa frase: «Hai coses y hai xente que tan avezaes a dir siempre n’ensame, en formación, en conxuntu y a comuña. Nun diben ser a facelo d'otra manera».

\footnotetext{
${ }^{1}$ En cántabru a cumuña, auzolan en euskaraz, roga en galego, treball a jova en català... En común, de manera conjunta, en comunidad... desde los trabajos comunales en las labores del campo hasta las redes vecinales que crean barrio en las ciudades. Hay personas, entornos, vidas, que sólo se pueden cuidar de manera grupal, en red.
} 
En efecto, hay cosas y hay gente tan habituadas a ir siempre en grupo, en formación, en conjunto y codo con codo, que no saben hacerlo de otra manera. Siempre en común.

Se nos ha parado la vida de golpe a todas a la vez, así, sin planificación ni espera previa, sin excepciones. Nos hemos visto encerradas en casa, como quien dice, de un día para otro y, lo que es más importante, lo hemos hecho sabiendo que la situación que nos atraviesa es la situación que atraviesa al mundo social en conjunto. Se nos ha impuesto un «distanciamiento social», buscando la solución del problema a través de la creación de una especie de antisociedad, donde nuestro mayor cometido ha sido aprender a estar sin estar, lo que, de forma indirecta, ha provocado que la distancia simbólica se acorte al mismo tiempo que se alarga la distancia física. Las ventanas y balcones se han convertido en espacios esenciales en nuestra vida, a los que nos hemos asomado para comprobar que el mundo sigue ahí y el sol sigue saliendo, aunque nosotras no lo hagamos, (re)conociendo el espacio que nos envuelve y a quienes lo habitan, descubriendo la vida que se esconde al otro lado de las ventanas que rodean a la nuestra. La lógica velocista que envuelve a las ciudades se ha roto y nos hemos parado a escuchar a nuestras vecinas, adquiriendo conciencia del otro, la otra, el otre, de sus preocupaciones, miedos y alegrías que, de alguna forma, también son las nuestras.

En este contexto han surgido distintas formas de organización ciudadana para el cuidado mutuo y la ayuda a los colectivos más vulnerables ante esta situación, creándose redes sin precedentes y extendiendo y fortaleciendo aún más las que ya existían. Esto, como señala Rebecca Solnit (2010), no es algo que se salga de lo común, sino que es frecuente que en situaciones excepcionales negativas para la población (como puede ser una catástrofe natural, un atentado o una pandemia) afloren los movimientos de ayuda mutua. En España las formas a través de las que se ha dado esa organización y las acciones que se han llevado a cabo han sido múltiples y diversas, desde la ayuda para cubrir necesidades básicas hasta iniciativas creativas que resaltan la importancia del reconocimiento de las emociones durante el confinamiento.

En los primeros días de la pandemia, cuando la situación empezaba a alejarse de la normalidad, pero sin ser todavía excepcional, comenzamos a ver iniciativas individuales de personas que se ofrecían para ayudar a las que tenían cerca, destacando sobre todo los carteles que aparecieron en algunos portales donde estudiantes se ofrecían a cuidar a los/ as niños/as del edificio si no había otra persona que pudiera hacerlo ante la cancelación de las clases. Estas iniciativas no han desaparecido con la formación de redes colectivas más amplias, sino que durante toda la pandemia nos han llegado, principalmente a través de redes sociales, historias de bloques de vecinas en los que se han generado formas de comunicación para saber si todas están bien, vecinas que le hacen la compra a otras más mayores que no pueden salir, que se ofrecen a ayudar en lo que necesiten a quienes continúan trabajando...

A medida que se puso en evidencia que la situación de excepcionalidad iba a continuar alargándose en el tiempo han ido surgiendo distintas iniciativas colectivas articuladas 
principalmente desde tres ámbitos: voluntariados organizados a través de ayuntamientos, páginas web y aplicaciones para conectar a la gente o promover iniciativas, y redes vecinales directas, que fueron las primeras en ponerse en marcha debido a que muchas funcionan sobre redes que existían de forma previa.

La principal forma a través de la que las personas se han puesto en contacto han sido las redes de solidaridad que se han formado en los barrios de numerosas ciudades y en algunos pueblos más pequeños. Estas se han extendido entre las vecinas a través de carteles en los portales, pancartas en los balcones, redes sociales y el boca a boca. Así, la diferencia entre el número de personas al que han llegado las distintas redes, teniendo mucho más alcance las que se sustentan en relaciones ya formadas como ha ocurrido con la Elkarlaguntza Sarea de Galdakao $^{2}$, ha puesto en evidencia la necesidad de formar redes barriales que puedan dar respuesta en los momentos de crisis. En Bilbao se ha formado una red de redes ${ }^{3}$ para organizar y compartir las iniciativas producidas en todos los barrios, las cuales se han centrado en su mayoría en la ayuda a personas vulnerables a través de realización de compras o mediación con los servicios sociales; el reparto de tarjetas SIM para facilitar la continuación con los estudios a quienes no tienen acceso a internet; la confección de batas y mascarillas para personal sanitario, residentes y comerciantes; la impresión 3D de viseras cubre rostro y piezas de respirador; y la organización de las donaciones de materiales necesarios para la realización del resto de actividades. Pero estas no son las únicas acciones que se están produciendo: también en Bilbao se han ofrecido personas voluntarias para hablar por teléfono en euskera con niños que no pueden practicar el idioma en sus casa; en Madrid el AMPA de alguno colegios se ha organizado para intentar suplir las carencias que deja la falta de comedores; hay quienes se han ofrecido para llamar y hacer compañía desde la distancia a personas mayores que están solas; en Otxarkoaga son jóvenes magrebíes de un albergue del barrio quienes realizan las compras a las personas mayores... Y a todas estas iniciativas se suman aquellas que tienen por objetivo cubrir las necesidades básicas de personas pertenecientes a los colectivos más vulnerados, algunos ejemplos de ellos son la Caja de Resistencia Antirracista ${ }^{4}$ impulsada por personas racializadas de Bilbao, la cesta solidaria de San Francisco, una iniciativa que recauda dinero para entregar cestas de comida a personas que aún no están siendo atendidas por los servicios sociales; o el fondo económico que está intentado reunir la Asociación de Mujeres Jóvenes Musulmanas residentes en Euskadi BIDAYA.

Las iniciativas de base también se han volcado en apoyar el comercio local y en el fomento de una agricultura que favorezca a los pequeños productores. Hechos como la falta de

\footnotetext{
2 En Galdakano existe una red de apoyo mutuo permanente que, a raíz de la COVID-19, ha creado una serie de redes y proyectos, algunas de ellas de en conjunto con el ayuntamiento, para ayudar a satisfacer las necesidades de las más afectadas. https://instagram.com/elkarlaguntza_sarea_galdakao?igshid=1drvsnlcd4v2n

${ }^{3}$ Debido a la multitud de iniciativas y redes barriales surgidas ante el confinamiento, se ha visto necesario crear una página web y una serie de canales de comunicación que conecten todas ellas y las hagan más accesibles a la población. https://www.elkartasunsareak.com/

${ }^{4}$ La Caja de Resistencia Antirracista es una iniciativa anónima y autogestionado que pretende servir de apoyo a aquellas personas atravesadas por una gran precariedad poniendo a su disposición dinero, alimentos u otro tipo de ayudas https://www.facebook.com/CAJA-resistencia-BILBO-102558504754730/
} 
material sanitario y el miedo inicial a que esta situación derivase en una falta de abastecimiento para toda la población han hecho presentes los problemas de la globalización, fortaleciendo la idea de que necesitamos una red de comercio local que nos garantice el abastecimiento cuando, en momentos como el actual, el transporte no sea posible, y en la que se propicie un mayor cuidado del planeta y de todas las personas que forman la cadena de producción. Además, el pequeño comercio de barrio ha sido fundamental en algunos lugares para impulsar y posibilitar las iniciativas que mencionábamos anteriormente y vertebrar comunidad, demostrando la importancia de la vinculación que este tiene con el barrio y con quienes lo habitan. Es por ello que en Bilbao se han difundido documentos durante toda la cuarentena con el nombre y la dirección de los comercios que seguían abiertos, y los comerciantes del Casco Viejo han elaborado un manifiesto fomentando el comercio local ante la desescalada; las y los Baserritarras de Bizkaia ${ }^{5}$ se han unido pidiendo la apertura de los mercados, señalando que estos forman su sustento de vida y la forma de propiciar una alimentación saludable y responsable a un gran número de personas; en Cantabria la plataforma "Cantabria no se vende» ${ }^{6}$ ha repartido cestas de apoyo al consumo local con productos que ahora no tienen salida en el mercado; en algunas partes de Sevilla y Madrid se ha reivindicado la importancia de los huertos urbanos como elementos claves para avanzar hacia el autoabastecimiento, y muchos han sido cedidos a personas migrantes en situación irregular...

Como ya hemos señalado, no todas las iniciativas han llegado con un rostro o una firma, desde la cercanía de los vecindarios, sino que internet ha tenido un papel clave a la hora de conseguir que las personas conozcan todas las alternativas que hay a su disposición, tanto para ofrecer ayuda como para ser ayudadas. Se han puesto en marcha diversas aplicaciones - como «la noria social hub» en Málaga o «¿tienes sal?» en Madrid, Barcelona y Valencia- con el objetivo de conectar a las personas cercanas; igualmente, se han creado páginas web (como SOLIVID o comoayudar.org) y bases de datos con listados y mapas de todas las iniciativas solidarias que se están llevando a cabo en determinados territorios.

Hay un lugar al que todas las iniciativas mencionadas no llegan, pero que tampoco se ha quedado vacío, el de las emociones. Además de los recursos materiales se vuelven básicos los emocionales, las prácticas que nos remueven por dentro desde el amor y el cariño, contribuyendo a nuestro bienestar. Necesitamos iniciativas que nos distraigan, que nos conecten con los sentimientos y nos ayuden a mirar el mundo desde el arte y la cultura, desde las cosas que «nos ablandan el corazón». Para ello son muchos los artistas que se han acercado de forma telemática a nuestras casas llenándolas de música, ilustraciones, actuaciones teatrales, magia, cuentos... También desde lo más cercano: calles con cuerdas

\footnotetext{
${ }^{5}$ https://ehkolektiboa.eus/merkatu-eta-azokak-irekita-nahi-ditugu/?fbclid=IwAR131Y_j-aPJt7F3-XhWhdCYZ3VJ_ rfytRf-cYhPywfUq-ocBy9Be46tW90

6 «Cantabria no se vende» es una iniciativa surgida en Cantabria con el objetivo de contribuir a crear una base social que plantee alternativas a los problemas sociales, económicos y políticos existentes en la Comunidad Autónoma. Esta iniciativa ha sido la que ha organizado la Red Cántabra de Apoyo Mutuo durante la pandemia y los distintos proyectos que surgen de ella. http://cantabrianosevende.org/red-cantabra-de-apoyo-mutuo
} 
llenas de color que van de un balcón a otro, arcoíris y carteles de ánimo en las ventanas, música por las tardes, niñas mandando dibujos a personas mayores, memes en internet... Ideas que surgen del reconocimiento de que la alegría también alimenta, aunque no dé de comer.

Todo esto nos lleva necesariamente a la reflexión de qué es eso de la solidaridad y cómo vamos a hacer que perdure en el tiempo. Podemos hablar de una supuesta solidaridad que se ejerce desde el poder, nutriendo el ego propio a través de la ayuda a los demás, y que opera a través de divisiones entre quienes ruegan ayuda y quienes tienen las condiciones materiales para ofrecerla; pero también de la solidaridad que entiende que todas somos profundamente interdependientes y que, frente a las divisiones, pugna por la construcción de un "nosotras». El problema de la solidaridad fuerte, "la buena», es que emana principalmente en contextos en los que los problemas son globales, donde al sentir el riesgo en nuestra propia piel intentamos paliar también el riesgo ajeno (quizá porque nunca es realmente ajeno), lo que hace que su prolongación en situaciones de «normalidad» sea menos asegurable. Se trata además de una solidaridad que se vuelve incompatible con las lógicas del capitalismo, no solo por el individualismo que este ensalza, sino también porque los ritmos de inmediatez que marca provocan que los tiempos del capital choquen de frente con los tiempos del cuidado, privándonos de la posibilidad, y de la energía, para pararnos a preguntarle a la vecina del quinto si necesita algo.

Por último, consideramos de especial importancia resaltar algunos de los problemas que atraviesan a la acción comunitaria. Al tratar esta de llegar a donde no llega el Estado, cubriendo los huecos que dejan las instituciones, corremos el riesgo de que se normalice que los movimientos sociales cubran necesidades que deberían garantizar las instituciones, pues, aunque esta organización es fundamental, hay sitios a los que no puede llegar, principalmente cuando hablamos de recursos económicos. Por ello, es importante que existan redes que den apoyo a los sectores que el Estado olvida, pero sin dejar de exigir y reivindicar que la respuesta a esos problemas tiene que venir desde el Gobierno, como está haciendo la campaña impulsada por diversas organizaciones exigiendo al gobierno un plan de Choque Social ${ }^{7}$. Además, si queremos que lo surgido ahora se fortalezca y extienda en el tiempo debemos mantener la defensa de lo común desde la desburocratización de los procesos, entendiendo que el acompañamiento debe darse de manera horizontal y desde la confianza, mostrándose como algo accesible a todo el mundo sin exigir una justificación.

\footnotetext{
${ }^{7}$ Las medidas que pide esta iniciativa se centran en tres ejes: suspensión del pago del alquiler, de la hipoteca y de los suministros básicos, para toda aquella persona que pierda ingresos; ningún despido y renta básica para quien se quede sin ingresos; e intervenir la sanidad privada sin que haya una posterior compensación económica.

Redacción El Salto (2020). «Organizaciones sociales exigen al Gobierno un Plan de Choque Social ante la crisis». El Salto, disponible en https://www.elsaltodiario.com/coronavirus/organizaciones-sociales-exigen-gobiernoplan-choque-social
} 


\section{CONCLUSIÓN: TEJIENDO REDES (IN)VISIBLES}

Nombrar las cosas, (re)definirlas, sentirlas, vivirlas, sufrirlas... disfrutar del proceso. Desde la experiencia propia y el (re)conocimiento de la ajena, desde el compartir. Tender lazos, tirar barreras, establecer conexiones, crear redes. Todo ello es necesario para entender el mundo que nos construye y ser capaces de construirlo, para entender todas las partes y comprender que su suma da más que el todo. Este es el objetivo que dirige la presente reflexión: analizar qué está ocurriendo, de dónde surgen sus raíces y cómo conectan entre sí todas las cosas que nos atraviesan.

Olvidar el cuerpo, dar por hecho su existencia y no preguntarnos qué necesidades lo recorren, qué y quién hace posible dicha existencia, es decir, cómo se satisfacen esas necesidades, nos lleva a ignorar la corporalidad de las personas y todos los límites que la rodean. El cuerpo pasa a verse únicamente como un objeto, un recipiente en el que se guarda la fuerza de trabajo, un medio para la obtención de una mayor acumulación de capital. Ignorando la conexión cuerpo-mente, que conectan porque son uno, y tratando el cuerpo como si fuera algo que se tiene, pero no se siente, no se es. Ignorar el cuerpo es ignorar la dimensión material de la vida y, sobre todo, es ignorar que la vida no se sostiene sola, sino que detrás hay todo un entramado de cuidados impidiendo que se caiga y, por tanto, un gran número de personas — casi siempre mujeres, casi siempre migrantes si hablamos de cuidados remunerados-, a las que tampoco se ve, realizando esta actividad.

Los cuidados engloban un sinfín de formas: podemos hablar de cuidados comunitarios, personales, de autocuidado, de cuidados forzosos y de cuidados mutuos... desde aquellos que son movidos por la remuneración económica hasta los basados en el desear el máximo bienestar - bienvivir - de las personas que queremos, pasando por múltiples y diversas casuísticas. Pero una parte de ellos, la primera en la que se centran las definiciones más limitadas, pasa por el cuidado del cuerpo y el entorno que le rodea, asociándolos con la realización de tareas del hogar o la atención a personas dependientes (cuando pensamos en cuidar a niñas y niños o a mayores solemos pensar en su soporte físico). Lo que conecta, de nuevo, los cuidados y el cuerpo y hace visible la estrecha relación entre la invisibilización de ambas esferas.

Sacar esto a la luz siempre ha sido importante, pero en este contexto, además de necesario, se vuelve inevitable. La situación generada por la COVID-19 ha convertido a la enfermedad en la protagonista de nuestras vidas, y con ella han florecido las conversaciones sobre el cuerpo al que afecta y los cuidados que intentan frenarla, así como las personas que los realizan. Hemos empezado a hablar del cuerpo, de qué consecuencias tiene la enfermedad en él, de la necesidad de mantener limpias nuestras manos, del peligro de tocarnos la cara... Toda la actividad política, económica y social ha comenzado a girar en torno a la enfermedad, los discursos en televisión y las noticias de prensa se han llenado de palabras como «contagios», «muertes», «anticuerpos», "grupos de riesgo», lo que nos ha mostrado 
que lo que afecta al cuerpo, afecta en mayor o menor medida al conjunto del sistema social. Todo ello vuelve innegable la necesidad de poner la vida en el centro.

La pandemia vivida ha dejado en evidencia las carencias de nuestra sociedad y su consecuente incapacidad para poder declararse como una sociedad que cuida, pero también se han producido ciertos hechos que nos han acercado a eso que podríamos considerar como una sociedad cuidadora. Desde aquí tenemos claro que una sociedad que cuida, escucha y conoce las distintas realidades que la componen, trabaja de forma horizontal para que las personas que cuidan también sean cuidadas, también narren y también decidan. Piensa «con» y no «sobre». Lo que implica conocer las necesidades de todas las personas, tanto las que se dan a nivel general — colectivo-, como las que se producen a nivel concreto, cambiando la concepción dicotómica que establece un margen y una periferia, un arriba y un abajo donde siempre hay gente que se queda fuera, por una concepción del mundo en red, un micelio, donde todas nos nutramos de todas. Todo esto también pasa por un Estado que sintamos que nos cuida, para lo que necesitamos políticas que se adapten a las realidades de la población, no que se planteen sobre situaciones abstractas en las que la mayoría de personas no encajan. Pasa por entender que necesitamos ayudas al cuidado porque vivimos en un país en el que la mayoría de las personas no pueden teletrabajar; que una renta mínima vital que no llega a todo el mundo no es suficiente cuando los ingresos de la mayoría de las familias han sufrido un descenso sin precedentes; que niños y jóvenes no son ajenos a lo que ocurre, por lo que su rendimiento académico no puede ser el mismo; que no es posible establecer un confinamiento obligatorio y olvidar a quienes no tienen casa; que las condiciones de reapertura no afectan igual a los pequeños comercios que a las grandes superficies...

Asimismo, reafirmamos que una sociedad que cuida es una sociedad comunitaria, partiendo de la idea de que el tejido social es imprescindible para entender que los problemas que nos afectan parten de raíces comunes, por lo que la búsqueda de soluciones debe ser colectiva y no individual. Las redes comunitarias fuertes son el mejor antídoto ante la soledad, al funcionar como punto de apoyo y no dejar a nadie atrás. Necesitamos construir espacios donde nos sintamos seguras y respaldadas, para lo que es imprescindible construir comunidad desde abajo, hacer nuestros los espacios que habitamos, sentir como hogar el barrio, el pueblo, la calle, en compañía de quienes lo comparten con nosotras, de quienes le dan vida, entendiendo que el cuidado del mundo empieza por el cuidado de lo local.

\section{BIBLIOGRAFÍA}

Addati, L., Cattaneo, U., Esquivel, V. and Valarino, I. (2018). Care work and care jobs for the future of decent work. Geneva : International Labour Office. https://www.ilo. org/wcmsp5/groups/public/---dgreports/---dcomm/---publ/documents/publication/ wcms_633135.pdf 
Aguiar, F., González, A. y Noguera, J.A. (2009). El giro analítico en Sociología. Revista Internacional de Sociología, n. ${ }^{\circ}$ 2, pp. 437-456.

Alabao, N. (2019). La crisis de cuidados, un debate pendiente. CTXT, 20 febrero. Disponible en https://ctxt.es/es/20190220/Firmas/24553/crisis-de-los-cuidados-dependenciafeminismo-residencias-de-ancianos-nuria-alabao.htm

Angelcos, N. (2016). Subjetividad, cuerpo y afecto en la teoría sociológica. Revista Estudios Avanzados, n. ${ }^{\circ}$ 26, pp. 76-94.

Alfonso, Í. (2020). Garamendi (CEOE). «El gobierno ha actuado con precipitación». Las mañanas de RNE. Disponible en https://www.rtve.es/noticias/20200330/ceoe-acusagobierno-precipitacion-falta-dialogo-decreto-parada-actividades/2011038.shtml

Álvarez Cantalapiedra, S. (2017). Capitalismo neoliberal y cuerpo. Papeles de relaciones ecosociales y cambio global, n. ${ }^{\circ}$ 137, pp. 5-10.

Anguita, J. (2020). Decía Gramsci. El viejo topo, 16 mayo. Disponible en https://www. elviejotopo.com/topoexpress/decia-gramsci/

Antón-Hurtado, F. (2012). Antropología del sinsentido. Revista de antropología experimental, n. ${ }^{\circ} 12$, pp. 349-371.

Asociación Libre de Abogadas y abogados (2020). Derechos de las personas trabajadoras del hogar durante la situación provocada por la pandemia del COVID-19 o coronavirus. Disponible en https://ala.org.es/wpcontent/uploads/2020/03/DERECHOS-DELAS-PERSONAS-TRABAJADORAS-DEL-HOGAR-DURANTE-LA-SITUACI\%C3\%93NPROVOCADA-POR-LA-PANDEMIA-DEL-COVID-19-O-CORONAVIRUS.-2.pdf

Blog Saltamontes (2020). Ayudas mutuas en tiempos de pandemia. El Salto, 24 abril. Disponible en https://www.elsaltodiario.com/saltamontes/ayudas-mutuas-en-tiempos-depandemia

Calle, E., González, J. y Saiz, M. (2020). Transformemos la solidaridad en pandemia. El Salto, 10 abril. Disponible en https:/www.elsaltodiario.com/mapas/ transformemos-la-solidaridad-en-pandemia

Candelas, S., Martín, C. y Cubilledo B. (2020). Vínculos significativos, o cómo hacer frente a la soledad no deseada en tiempos de confinamiento. Público, 8 mayo. Disponible en https://blogs.publico.es/otrasmiradas/32748/vinculos-significativos-o-como-hacerfrente-a-la-soledad-no-deseada-en-tiempos-de-confinamiento/ 
Carrasco, C. (2011). La economía del cuidado: planteamiento actual y desafíos pendientes. Revista de Economía Crítica, n. ${ }^{0}$ 11, pp. 205-225. http://revistaeconomiacritica.org/ sites/default/files/REC11_9_intervenciones_CristinaCarrasco.pdf

Carrasco, C. (2013). El cuidado como eje vertebrador de una nueva economía. Cuadernos de relaciones laborales, Vol. 31, n. ${ }^{0} 1$, pp. 39-56. http://dx.doi.org/10.5209/rev_ CRLA.2013.v31.n1.41627

Carrasco, C. (2017). La economía feminista. Un recorrido a través del concepto de reproducción. Ekonomiaz, n. ${ }^{\circ}$ 91, pp. 52-77.

Carrasco, C., Borderías, C. y Torns, T. (eds.) (2019). El trabajo de cuidados: historia, teoría y políticas. Madrid: Los Libros de la Catarata.

CERMI (2020). El CERMI denuncia el «fracaso» del Sistema de Autonomía y Atención a la Dependencia y exige su «refundación». 5 de octubre. https://www.cermi.es/es/ actualidad/noticias/el-cermi-denuncia-el-\%E2\%80\%9Cfracaso $\%$ E2\%80\%9D-delsistema-de-autonom\%C3\%ADa-y-atenci\%C3\%B3n-la

Clemente, Y., Grasso, D., Blanco, P.R., Sevillano, L. y Zafra, M. (2020). Casos confirmados de coronavirus en España y en el mundo. El País, 4 noviembre. Disponible en https:// elpais.com/sociedad/2020/07/27/actualidad/1595838623_808240.html

Coffey, C., Espinoza, P., Harvey, R., Lawson, M., Parvez Butt, A., Piaget, K. y Sarosiy Julie Thekkudan, D. (2020). Tiempo para el cuidado. El trabajo de cuidados y la crisis global de desigualdad. Oxford: Oxfam Internacional. https://aipaz.org/wp-content/ uploads/2020/01/tiempo-para-cuidado-informe-davos-2020.pdf

Del Rio, C., Collins, L.F. y Malani, P. (2020). Long-term Health Consequences of COVID-19. JAMA, 324(17), pp.1723-1724. doi:10.1001/jama.2020.19719

Ecuador etxea (2020). Jóvenes musulmanas en Euskadi crean campaña de supervivencia ante la situación de crisis. Ecuador etxea. Disponible en https://www.ecuadoretxea. org/jovenes-musulmanas-en-euskadi-crean-campana-de-supervivencia-ante-lasituacion-de-crisis/

Ezquerra, S. (2011). Crisis de los cuidados y crisis sistémica: la reproducción como pilar de la economía llamada real. Investigaciones Feministas, vol. 2, pp. 175-194. http:// dx.doi.org/10.5209/rev_INFE.2011.v2.38610

Farré, L. y González, L. (2020). ¿Quién se encarga de las tareas domésticas durante el confinamiento? Covid-19, mercado de trabajo y uso del tiempo en el hogar». Nada es Gratis, 23 de abril. https://nadaesgratis.es/admin/quien-se-encarga-de-las-tareas-domesticas 
Grupo de trabajo Ética y Covid Euskadi (2020. ¿La salud de quién estamos defendiendo? Desigualdades sociales y sanitarias en tiempo de pandemia, 6 de junio. Disponible en http://www.asociacionbioetica.com/uploads/files/Desigualdades $\% 20$ sociales $\% 20$ y\%20sanitarias\%20en\%20tiempos\%20de\%20Pandemia_\%20Versio\%cC\%81n\%20 completa.pdf

Le Breton, D. (2018). La Sociología del Cuerpo. Madrid: Siruela.

Lizón, A. (2010). Encrucijadas teóricas en la sociología del siglo xx. Papers, vol. 95, n. ${ }^{\circ}$ 2, pp. 389-420. http://dx.doi.org/10.5565/rev/papers/v95n2.75

Lobo, M. (2019). A Comuña. Gijón-Xixón: Impronta.

Mugarik Gabe (2020). «Solidaridad que atraviesa fronteras». Pikara Magazine, disponible en https://www.pikaramagazine.com/2020/03/solidaridad-que-atraviesa-paredes

Oliva Portolés, A. (2015).: Hacia una ontología social del cuerpo en Butler: análisis y límites. Investigaciones Feministas, vol. 6, pp. 85-107. http://dx.doi.org/10.5209/rev_ INFE.2015.v6.51381

Pérez Orozco, A. (2006). Amenaza tormenta: la crisis de los cuidados y la reorganización del sistema económico. Revista de economía crítica, n. ${ }^{\circ}$, pp. 7-37.

Redacción El Salto (2020). «Preguntas y respuestas para las empleadas del hogar ante un decreto que no las contempla». El Salto [En línea], disponible en https://www. elsaltodiario.com/coronavirus/preguntas-respuestas-derechos-laborales-empleadashogar-no-contemplados-medidas-gobierno

Sáenz, M., Prieto, S., Moore, C., Cortés, L., Espitia, A. y Duarte, L. (2017). Género, cuerpo, poder y resistencia. Un diálogo crítico con Judith Butler. Estudios Políticos, n. ${ }^{\circ}$ 50, pp. 82-99. DOI: 10.17533/udea.espo.n50a05

Solnit, R. (2010). A paradise built in hell: The extraordinary communities that arise in disaster. New York: Penguin.

Tronto, J. (2005). Cuando la ciudadanía se cuida: una paradoja neoliberal del bienestar y le desigualdad. En Congreso Internacional Sare 2004: ¿Hacia qué modelo de ciudadanía? (pp. 231-253). Vitoria-Gasteiz: Emakunde. https://www.emakunde.euskadi.eus/ contenidos/informacion/publicaciones_jornadas/es_emakunde/adjuntos/sare2004_ es.pdf

Weil, S. (2014). La condición obrera. Traducción de Teresa Escartín Carasol y José Luis Escartín Carasol. Madrid: Trotta. 